\title{
Active Chemical Sampling System for Underwater Chemical Source Localization
}

\author{
Ryuichi Takemura, ${ }^{1}$ Hiromi Sakata, ${ }^{2}$ and Hiroshi Ishida ${ }^{1}$ \\ ${ }^{1}$ Graduate School of Bio-Applications and Systems Engineering, Tokyo University of Agriculture and Technology, Tokyo 184-8588, Japan \\ ${ }^{2}$ Department of Physical Electronics, Tokyo Institute of Technology, Tokyo 152-8552, Japan \\ Correspondence should be addressed to Hiroshi Ishida; h_ishida@cc.tuat.ac.jp
}

Received 16 October 2015; Revised 25 January 2016; Accepted 31 January 2016

Academic Editor: Alberto J. Palma

Copyright (C) 2016 Ryuichi Takemura et al. This is an open access article distributed under the Creative Commons Attribution License, which permits unrestricted use, distribution, and reproduction in any medium, provided the original work is properly cited.

\begin{abstract}
This paper investigates the effect of active water sampling to enhance chemical reception for small underwater robots. The search for a chemical source in a stagnant water environment is not an easy task because the chemical solution released from the source stays in the close vicinity of the source. No signal is obtained even if a robot with chemical sensors is placed a few centimeters from the chemical source. In the system under study, four electrochemical sensors are aligned in front of a suction pipe that draws water samples from the surroundings. Owing to the smooth laminar flow converging to the suction port, the streak of the chemical solution drawn to the sensors is shaped into a thin filamentous form. To prevent the chemical solution from passing between the sensors without touching their surfaces, slits are placed in front of the sensors to guide the incoming chemical solution from different directions to the corresponding sensors. A chemical source can be located by moving the system in the direction of the sensor showing the largest response. It is also shown that the chemical reception at the sensors can be significantly enhanced when the system is wobbled to introduce disturbances.
\end{abstract}

\section{Introduction}

Over the years, robots with chemical sensing capabilities have been developed for localizing underwater chemical sources [1-4]. Potential applications of such robots include searches for underwater wreckage and environmental pollutant sources. It is also expected that the robots will be applied to tracking of explosives dissolved into water from unexploded ordnance if highly sensitive chemical sensors for explosive detection are provided. One of the challenges for such chemical sensing robots is how to increase the chemical reception at the sensors [4]. Most types of chemical sensors show response only when chemical substances are actually brought to the surfaces of the sensors. The molecular diffusion in water is extremely slow, and the characteristic diffusion length in one hour is calculated to be $5 \mathrm{~mm}$ [5]. The number of chemical molecules that reach the surface of a chemical sensor on a mobile robot significantly decreases when the position of the robot is even slightly off from the distribution of the chemical substance drifting in water. To detect the chemical substance, we need to bring the sensor to the location of the chemical substance or to make some efforts to actively collect the chemical substance to the sensor surface.

Therefore, search for a chemical source in a stagnant underwater environment is not so easy as it appears to be. In rivers or tides, the velocities of water currents dominate the slow molecular diffusion. The chemical substance released from its source is widely dispersed in the environment by being transported by water currents. The chance of detecting the chemical substance is high in such an environment, and the robot can track the distribution of the detected chemical to its source. On the contrary, most of the chemical substance released from the source in stagnant water stays in the confined area around the source location. Therefore, a chemical sensor on a mobile robot would show no response even if the robot is just a few centimeters off from the location of the chemical source.

Despite the difficulty in obtaining chemical information in aquatic environments, many aquatic animals rely on their olfaction when searching for food [5-8]. An interesting 
example is foraging behavior of crayfish $[9,10]$. Most species of crayfish live in still water environments, for example, at the bottom of lakes or ponds where the velocity of water flow rarely exceeds $10 \mathrm{~mm} / \mathrm{s}$ [9]. A crayfish has three pairs of maxillipeds around its mouth below the antennules, and the flagella of these maxillipeds have a fan-like shape. The water currents generated by waving the maxillipeds are considered to help collect water samples from the surroundings to the chemoreceptors on the antennules. This type of active collection of water samples is observed for many aquatic animals including copepod [11], squilla [12], and some fish [13]. Although the ways of flow generation are more or less different among spices, these behaviors may be playing an important role to enhance the chemical detection by the aquatic animals.

The aim of our research presented in this paper is to investigate the effects of active collection of water samples for underwater chemical sensing. There have been extensive studies on olfactory mediated search behavior of animals in high Reynolds number turbulent flow, and various attempts have been made to develop bioinspired robots for such environments [1-3, 5-8]. The biggest challenge was how to cope with the random and chaotic nature of turbulent odor plumes. Our focus here, on the other hand, is on stagnant water environments. This paper shows that chemical source localization in stagnant water is not so straightforward as it appears to be, because of the highly confined chemical distribution in such an environment. We consider our work as an initial step toward obtaining better understandings on the behavioral implications of active odor perception by animals and also toward developing better design methodologies for underwater robots with chemical sensing capabilities. We have previously developed an underwater robot that mimics active water current generation by crayfish [4]. The robot is equipped with a pair of fanning arms that mimic the crayfish maxillipeds. By waving these arms, outward water currents are generated. Then, the inflow converging to the robot is induced by a physical phenomenon called jet entrainment [10], and water samples were drawn from the surroundings to the chemical sensors on the robot.

Here, we report on an underwater robotic system that collects water samples using a suction pump. Compared to the inflow generated by jet entrainment, the velocity of the flow generated by suction decays more rapidly with the distance from the suction port [10]. Therefore, suction may not be the most efficient way of collecting water samples. Suction feeding in the nurse shark, for example, is reported to be effective only within $3 \mathrm{~cm}$ from the mouth [14]. However, active sampling using a pump is of practical use because to mount a pump on a mobile robot is generally much easier than to equip the robot with small fanning arms. We have targeted stagnant water environments and tested the capability of our robotic system for chemical detection and source localization in a still water aquarium. Although such a low velocity flow field is not often seen in practical applications, a small benthic robot, for example, a lobster robot reported in [15], in search of small unexploded ordnance will encounter similar flow conditions. Moreover, how animals, for example, crayfish, accomplish their olfactory search in stagnant environments is

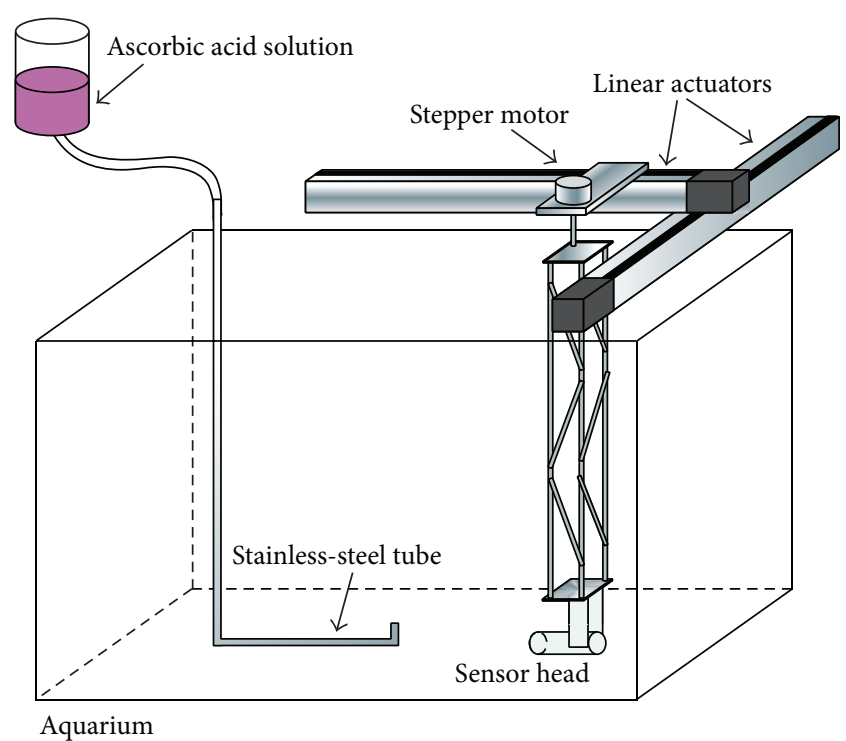

FIGURE 1: Schematic diagram of the active sampling system and the experimental setup.

of biological interest $[9,10]$. In the prototype system reported in this paper, four working electrodes of amperometric electrochemical sensors were aligned at the suction port. Despite the simplicity of this suction system, several technical aspects that need to be considered when designing such an active sensing system are clarified through the experiments. For example, our initial experiments showed that a chemical solution drawn from the surroundings to the suction port often passes between the sensors without touching their surfaces. Therefore, slits were placed in front of the working electrodes of the sensors. Each slit opening is aligned to each electrode to guide the incoming water samples from different directions to the corresponding sensor electrodes. It is shown that the angular detection range of each sensor electrode can be adjusted by changing the width of each slit. Experimental results are also presented to show that the reception of the incoming chemical solution at the sensors can be significantly enhanced by wobbling action of the sensing system.

\section{System Configuration and Experimental Setup}

The schematic diagram of the active chemical sampling system and the experimental setup is shown in Figure 1. The robotic chemical sensing system investigated in this research consists of three parts: a pump, a set of actuators, and a sensor head suspended from the actuators. Two linear actuators (petit-SERVO, THK Co., Ltd.) and a stepper motor (PF35T48C1, Nippon Pulse Motor Co., Ltd.) were used to move the sensor head in a Plexiglas water tank. As shown in Figure 1, the second linear actuator was mounted at a right angle on the moving stage of the first actuator. The stepper motor was attached to the moving stage of the second linear actuator, and the sensor head was suspended from the motor shaft. 


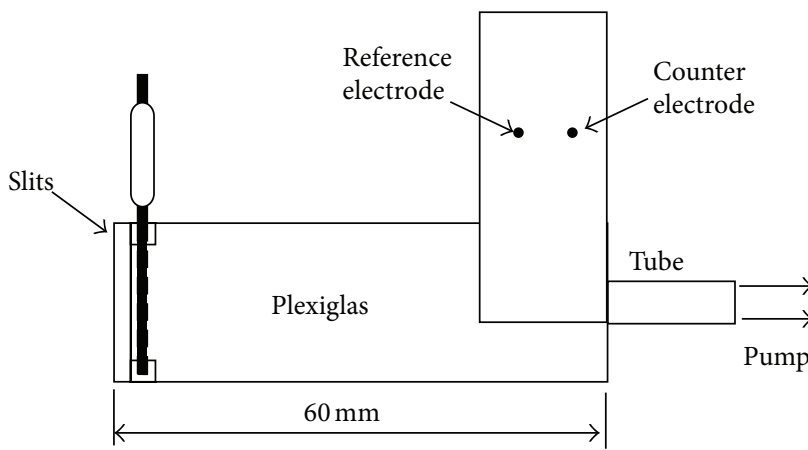

(a)

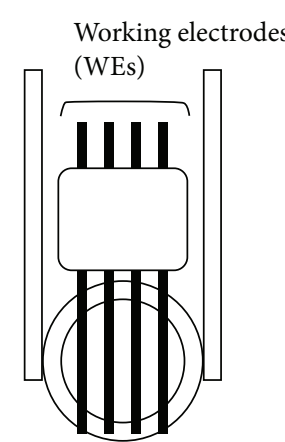

(b)

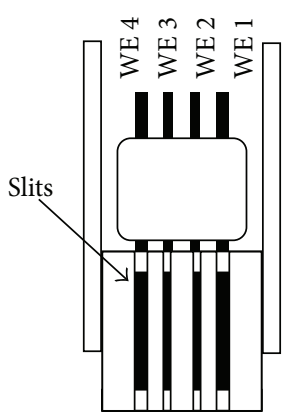

(c)

Figure 2: Schematic diagram of the sensor head. (a) Side view. (b) Front view without slits. (c) Front view with slits.

This setup allowed us to move the sensor head to any twodimensional coordinates in the aquarium in the specified orientation.

The schematic diagram of the sensor head is shown in Figure 2. The water pump (PMS-411B, Sanso Electric Co., Ltd.) was connected to a $60 \mathrm{~mm}$ long Plexiglas pipe with an inner diameter of $16 \mathrm{~mm}$ and an outer diameter of $19 \mathrm{~mm}$, through the tube at the right end of the Plexiglas pipe in Figure 2(a). Water samples were sucked from the surroundings into the left opening of the pipe, and the suction rate of the pump was adjusted to $1200 \mathrm{~mL} / \mathrm{min}$. The water sucked into the pump was drained to prevent the flow field in the aquarium from being disturbed by returning the water in the tank.

In the experiments, the Plexiglas aquarium $(300 \mathrm{~mm}$ wide $\times 600 \mathrm{~mm}$ long $\times 350 \mathrm{~mm}$ high) was filled with $0.485 \mathrm{M}$ salt water up to a depth of $50 \mathrm{~mm}$. The sodium chloride served as a supporting electrolyte for the amperometric electrochemical sensing, and its concentration was matched to the salinity of seawater. $10 \mathrm{mM}$ ascorbic acid solution was used as the detection target and was released from a stainlesssteel tube with an outer diameter of $1.61 \mathrm{~mm}$. The release rate was adjusted to $0.3-0.5 \mathrm{~mL} / \mathrm{min}$ by using a pinch valve. The outlet of the tube was placed at a height of $20 \mathrm{~mm}$ from the bottom of the aquarium and was pointed upward. $100 \mathrm{mg} / \mathrm{L}$ fluorescent dye (rhodamine 6G) was added to the ascorbic acid solution to enable visual observation of the solution released in the aquarium. Rhodamine $6 \mathrm{G}$ was shown to have no adverse effect on the electrochemical detection of the ascorbic acid [16]. The density of the solution was carefully matched to the salt water in the aquarium by adding a small amount of salt or water in the solution.

Most animals determine the direction of an odor source from the direction of the flow that carries the odor. Many animals locate an odor source by moving upstream when they are stimulated by food smell $[7,8]$. In stagnant water environments, however, you have to rely more on chemical signals than on the flow direction when determining the location of an odor source. The primary olfactory organs of crayfish are the pair of antennules. As shown in Figure 2, four carbon rods with a diameter of $0.9 \mathrm{~mm}$ were aligned laterally at an interval of $3.5 \mathrm{~mm}$ at the suction port of the Plexiglas pipe. Each carbon rod was connected to a custom-made potentiostat circuit and served as a working electrode (WE) for the electrochemical detection of ascorbic acid. Silver wire and a stainless-steel tube were used as a reference electrode and a counter electrode, respectively. The potential of the four working electrodes were kept at $0.7 \mathrm{~V}$ against the reference electrode [16]. The response of this electrochemical sensor system is the electrical current induced by the oxidation of ascorbic acid. The electrical currents from the four working electrodes were measured at a $100 \mathrm{~Hz}$ sampling rate. In the chemical source localization experiments in which the actuators were used to move the sensor head, the average values of the sensor response currents over $0.2 \mathrm{~s}$ were recorded at $5 \mathrm{~Hz}$ to eliminate the noise induced by the actuators. Slits were placed in front of the carbon electrodes to guide the incoming water samples from different directions to the corresponding electrodes. The direction of the chemical source was determined by comparing the averaged values.

To show the difficulty of chemical detection in a stagnant water environment, we first measured the response of an electrochemical sensor without using the pump to actively draw water samples from the surroundings. In this experiment, a single carbon rod electrode, instead of the sensor head, was suspended from the actuator system together with a silver reference electrode and a stainless-steel counter electrode. To measure the chemical distribution around its source, the set of electrodes was moved to scan a $70 \mathrm{~mm}$ by $70 \mathrm{~mm}$ area. The coordinate system and the scanning path were defined as shown in Figure 3. The chemical source was placed at $(x, y)=$ ( $45 \mathrm{~mm}, 35 \mathrm{~mm})$. In this experiment, the ascorbic acid solution was released from a porous glass ball to make the solution spread almost isotropically from the release point. The release was stopped when the released solution had spread to a diameter of $15 \mathrm{~mm}$. The tip of the carbon electrode was set at the same height as the chemical source ( $20 \mathrm{~mm}$ from the bottom). The scan was started from the origin of the coordinate system, and the electrodes were moved at $10 \mathrm{~mm} / \mathrm{s}$ along the path shown in Figure 3. The measured sensor response is shown in Figure 4. A large current was obtained only when the carbon electrode was brought within the proximity of the chemical source. Even when the electrode was only $20 \mathrm{~mm}$ from the source, almost no chemical was detected. This result suggests 


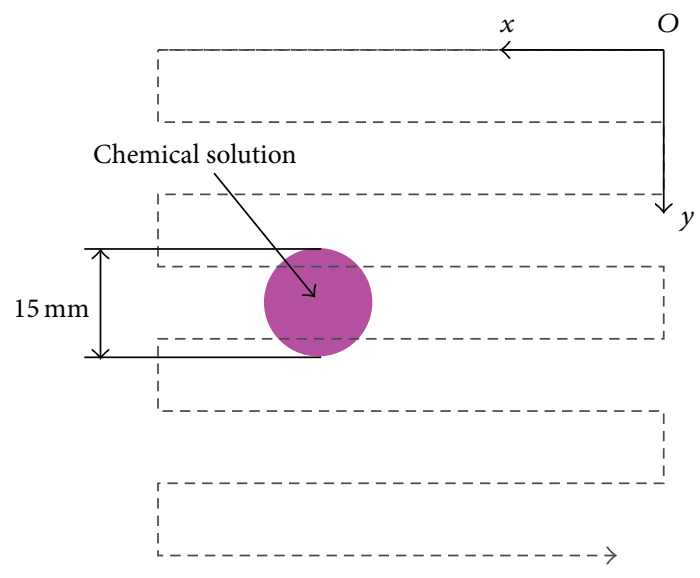

FIGURE 3: Scanning path of the sensor electrode. Ascorbic acid solution was released from $(x, y)=(45 \mathrm{~mm}, 35 \mathrm{~mm})$.

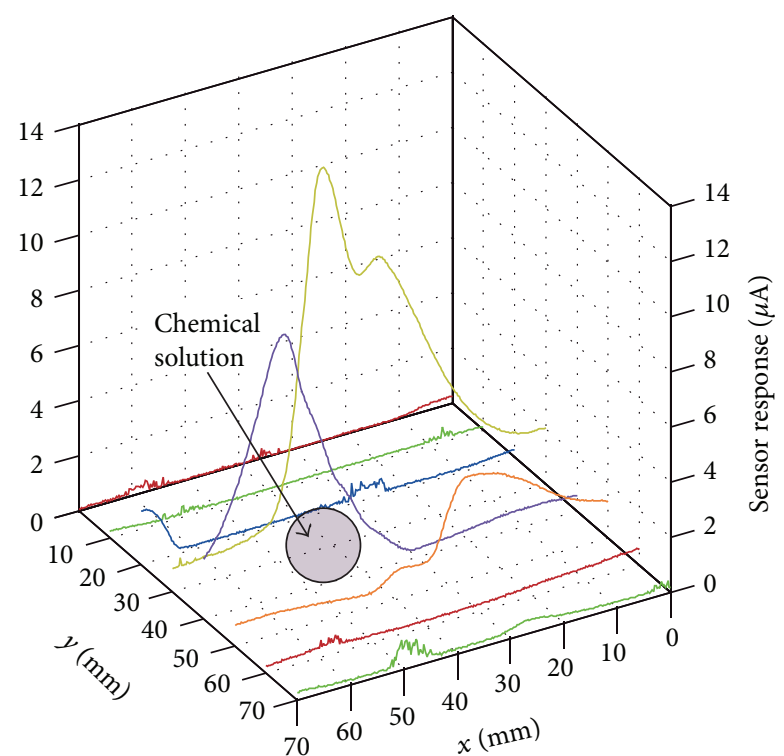

FIGURE 4: Sensor response current obtained along the scanning path shown in Figure 3.

that an underwater robot (or an aquatic animal searching for the smell of prey) may not notice the presence of a chemical source until it comes in the close vicinity.

\section{Chemical Detection Enhancement by Water Suction and Slits}

3.1. Effect of Slits on Chemical Detection. To investigate the effectiveness of active chemical sampling in a stagnant water environment, chemical detection trials were first conducted without placing the slits in front of the carbon working electrodes. However, the results were not successful. In each trial, the chemical source was placed at one of the 15 different locations shown in Figure 5. After waiting for the water currents in the aquarium induced by placing the chemical source and the sensor head to cease, the release of the chemical solution

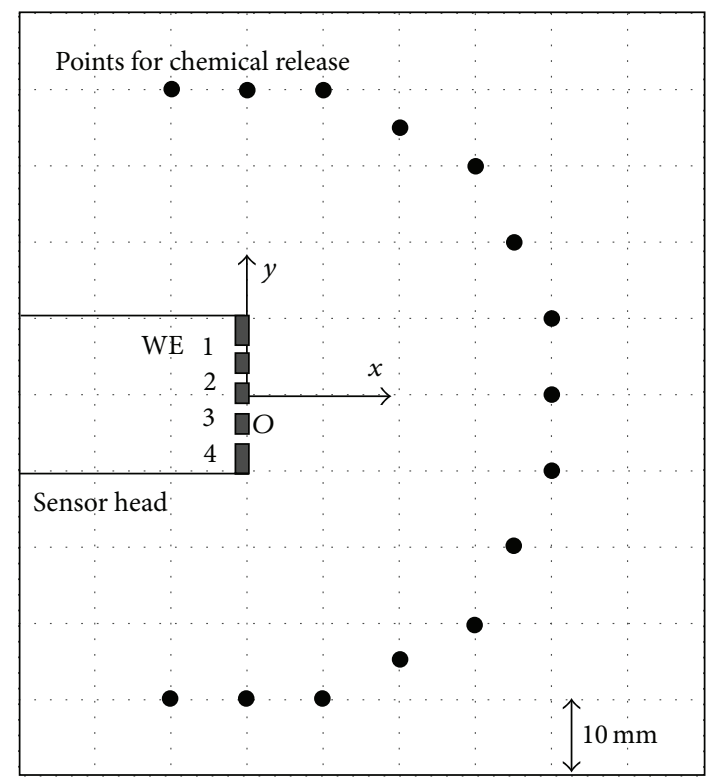

FIGURE 5: Top view of the sensor head and points for chemical release. The working electrodes were numbered from 1 to 4 from the left side of the sensor head.

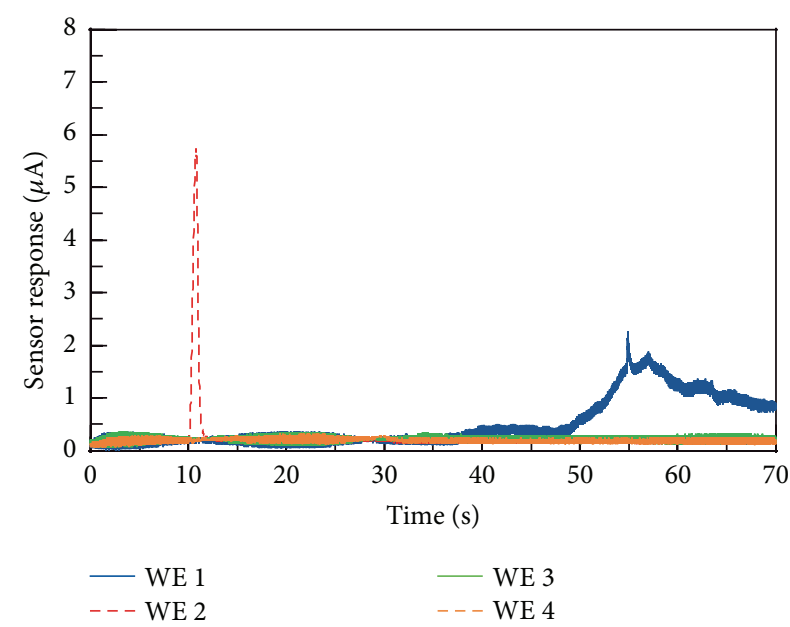

FIgURE 6: Typical sensor response curves observed when the slit was removed from the sensor head. The chemical source was placed at $(x, y)=(10 \mathrm{~mm}, 40 \mathrm{~mm})$.

and the recording of the sensor responses were started. After $5 \mathrm{~s}$ from the start of the chemical release, the pump was activated to suck water samples. By then, a blob of the chemical solution with a diameter of approximately $3 \mathrm{~mm}$ was formed at the chemical source. When the pump was activated, this blob was drawn to the suction port of the sensor head and was followed by a narrower tail of the chemical solution generated by the continuous chemical release from the source.

Figure 6 shows the typical sensor response curves observed in the chemical detection trials. The chemical source was placed at $(x, y)=(10 \mathrm{~mm}, 40 \mathrm{~mm})$ in the coordinate system shown in Figure 5 . When the blob of the chemical solution arrived at the suction port of the sensor 


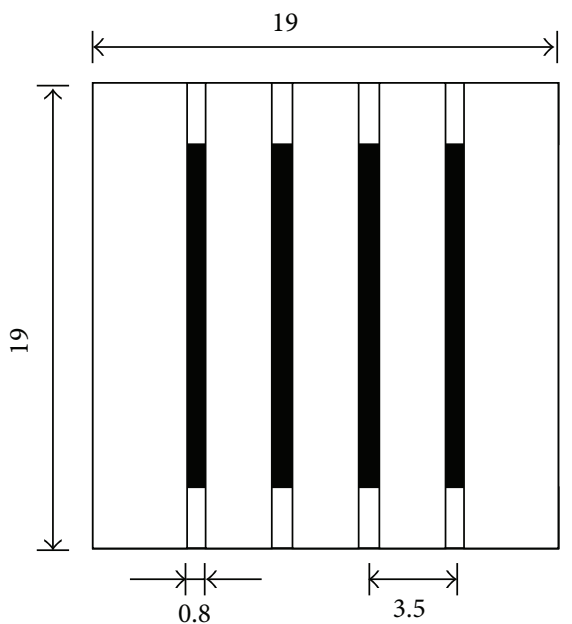

(a)

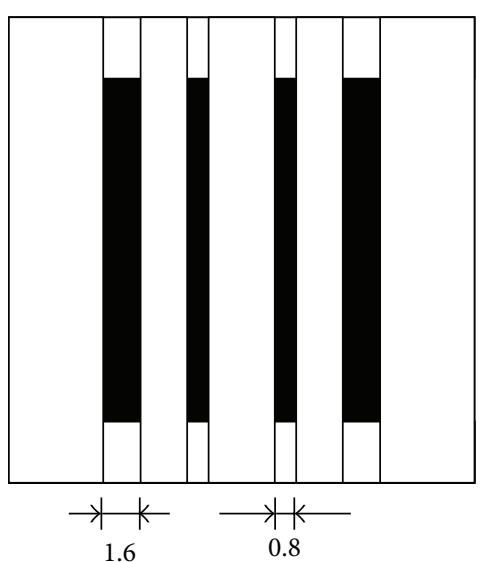

(b)

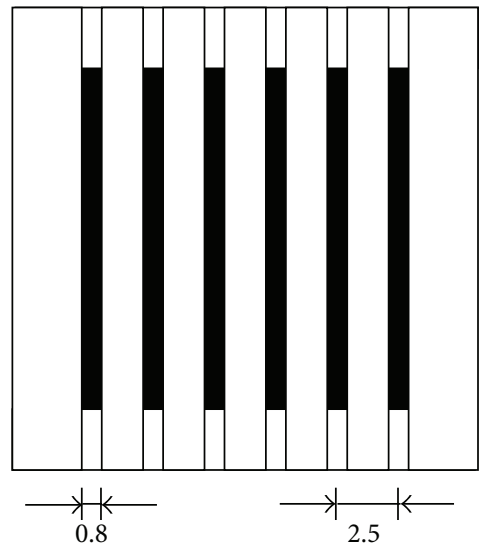

(c)

FIgURE 7: Schematic diagrams of the slit plates. Units are in millimeters. (a) Four equally spaced slits with 0.8 mm width. (b) Two wider outer slits with $1.6 \mathrm{~mm}$ width and two narrower inner slits with $0.8 \mathrm{~mm}$ width. (c) Six equally spaced slits with $0.8 \mathrm{~mm}$ width.

head, the blob hit the second carbon working electrode (WE 2). Thus, a large current response was obtained from WE 2 at $10 \mathrm{~s}$ from the start of the measurement ( $5 \mathrm{~s}$ after the activation of the pump). However, the tail of the blob passed between WEs 1 and 2 without touching them, and therefore, almost no sensor response was observed thereafter. The tail behind the blob was narrow because a steady laminar flow field was generated by sucking water. Only occasionally, fluctuations in the flow field made the tail meander and touch the sensor electrodes. It was confirmed from the visual observation of the chemical distribution that the large current from WE 1 from $50 \mathrm{~s}$ to the end of the recording was caused by such fluctuations. Among the 15 chemical source locations shown in Figure 5, the sensor response curves similar to Figure 6 were observed at eight locations. For the other seven locations, even the wider blob head passed between the electrodes.

To improve the chemical detection at the sensor electrodes, slits were placed in front of them as shown in Figure 2(c). The slits guide the water samples drawn from the surroundings to the sensor electrodes and reduce the probability of the streak of chemical solution passing between the electrodes. Three slit plates with different slit numbers and widths as shown in Figure 7 were tested. Slit plate (a) has four slits of equal width. The two outer slits of slit plate (b) are wider than the others. Slit plate (c) has six slits with equal width. These slit plates were fabricated by making saw cuts to a $3 \mathrm{~mm}$ thick Plexiglas plate. The fabricated slit plate was placed $1.5 \mathrm{~mm}$ in front of the working electrodes as shown in Figure 2(a), and the position of each working electrode was adjusted to the center of each slit. Typical sensor response curves obtained with the slit plate are shown in Figure 8. Slit plate (b) was used in this trial, and the chemical source was placed at the same location as in the trial shown in Figure 6. The small current peak appeared at $13 \mathrm{~s}$ when the blob of the chemical solution arrived at the suction port of the sensor head. This time, however, large response currents continued

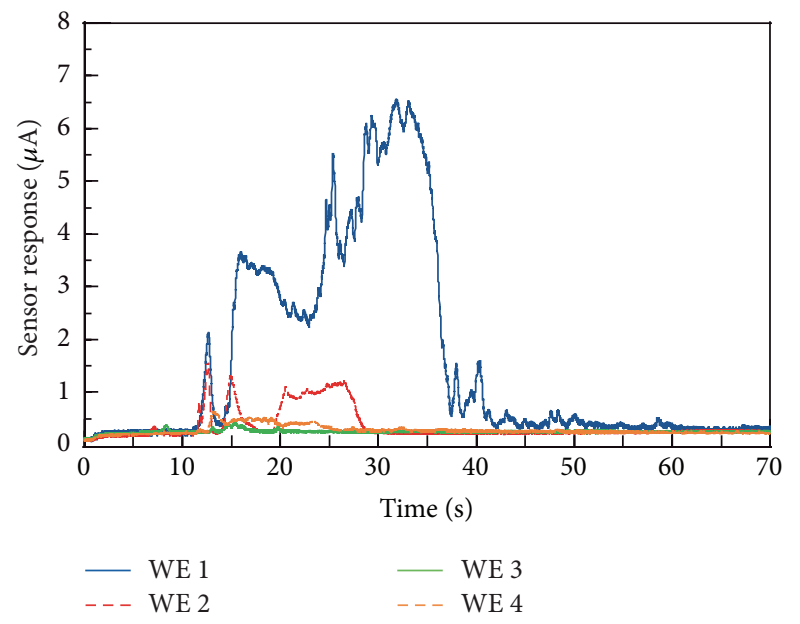

FIGURE 8: Sensor response curves obtained with slit plate (b). The chemical source was placed at $(x, y)=(10 \mathrm{~mm}, 40 \mathrm{~mm})$.

to be observed even after the wide blob head had passed and its narrower tail had arrived. Since the chemical source was placed on the left side of the sensor head, the leftmost electrode (WE 1) showed the largest response. The large sensor responses were obtained for all 15 chemical source locations tested. The sensor responses continuously exceeding $1 \mu \mathrm{A}$ for at least $20 \mathrm{~s}$ were obtained for 11 locations. For the other four locations, the sensor responses of similar magnitudes were obtained but they were more intermittent. Since the water samples from different directions were sucked into different slits, a large response current was most frequently obtained from the electrode closest to the chemical source.

The suction system under investigation here has some similarity to mammalian sniffing. By sniffing the air or water into nostrils, the sampling volume entering the nostrils is increased [17]. Moreover, the increased flow velocity over 
or inside the olfactory organ reduces the thickness of the boundary layer, and therefore, molecular diffusion to the surface of the olfactory organ through the boundary layer is promoted [17]. However, even if the odor is sucked into the nostrils and the thickness of the boundary layer is reduced, no signal can be obtained if the flow path of the sucked odor passes far outside the boundary layer of the olfactory epithelium. This issue becomes critical when trying to detect a chemical substance in water because the characteristic diffusion length in water is 100 times smaller than that in air. The sensor response curves obtained with the slits illustrate the importance of ensuring the contact of the sucked water sample with the sensor surface (or at least the contact with the outer perimeter of the boundary layer around the sensor electrode). The slits might be analogous to the complicated but sophisticated structure in the mammalian nasal cavity that guides the sucked air along specific paths for sensitive odor detection [17].

3.2. Adjusting Slit Widths. The three slit plates shown in Figure 7 were compared to see the effects of the number of the slits and their widths on the chemical detection at the sensors. To observe the suction of water samples into the slits, black ink was released from the stainless-steel tube that was used as the chemical source in the experiments described in the previous section. The ink was diluted with salt water to make it neutrally buoyant in the $0.485 \mathrm{M}$ salt water in the aquarium. Again, 15 chemical source locations shown in Figure 5 were tested. When the pump was switched on, the ink was drawn from the outlet of the stainless-steel tube to the slits. We observed the path of the ink to see into which slit the ink was drawn. The results are summarized in Figure 9. Each zone marked with a different pattern represents the area from which water samples are sucked into the corresponding slit. For example, Figure 9(a) shows that the ink released from the five topmost locations was drawn into slit 1 and guided to WE 1. The ink released from the next three locations was drawn to WE 2. Thus, each zone defines the angular range of chemical reception at each sensor. It should be noted that when the pump was activated, water samples were drawn not only from the front side of the sensor head but also from a wide angular range. Even the water on the backside of the sensor head was drawn into the outer slits.

Figure 9 indicates that the range from which water samples were drawn to each slit can be adjusted by changing the number of slits and their widths. Compared to Figure 9(a), water samples only in the narrower frontal regions are drawn to the inner slits (slits 2 and 3) in Figure 9(b) because the outer slits of slit plate (b) in Figure 7 were wider than its inner slits. This means that the direction of a chemical source can be determined with a higher angular resolution when using slit plate (b) compared to using slit plate (a). When slit plate (b) is used and the largest response current is obtained from WE 2, for example, the source can be determined to be in the approximately 30 -degree range in front of slit 2 . When slit plate (a) is used, the directional ambiguity increases. The direction of the chemical source is determined only to be in the 45-degree range in front of slit 2 . The higher angular resolution in the front is obtained in exchange for the lower resolution on the sides. Nevertheless, using slit plate (b) is beneficial for chemical source localization. Once the direction of a chemical source is roughly determined and the search agent starts proceeding toward the source, the agent often needs to fine-tune its course rather than making a large turn. Therefore, slit plate (b) was used in the chemical source localization experiments described in the following sections. If you can afford to have more sensors on your robot or sensing system, higher angular resolution in the determination of the chemical source direction can be also attained by increasing the number of slits as shown in Figure 9(c).

\section{Chemical Source Localization Experiments}

The chemical source localization experiments were conducted by moving the sensor head toward the direction of a chemical source determined from the responses of the amperometric sensors. The coordinate system was defined in the aquarium as shown in Figure 10. The origin of the coordinate system was set near the center of the aquarium. The sensor head was initially positioned at the origin of this coordinate system, as shown in Figure 10. Then, the pump was activated to collect water samples to the sensors. The sensor head was kept at the same position until at least one of the sensor response currents exceeded a threshold value. Then, the sensor head was moved toward the direction of the most responding sensor. The threshold value was set by multiplying the average sensor response currents for $5 \mathrm{~s}$ before the start of the first trial of the day by 3.8. When all response currents dropped below the threshold, the sensor head was stopped to wait for a chemical substance to be drawn to the sensors.

The translational and rotational velocities of the sensor head were set to $5 \mathrm{~mm} / \mathrm{s}$ and $36 \mathrm{deg} / \mathrm{s}$, respectively. These values were empirically adjusted to make sure that the chemical solution released from the source was continuously drawn to the sensor head. If the sensor head was moved too fast, it was required to wait for a while for the chemical solution to be drawn to the new location of the sensor head. The turning angles were determined based on Figure 9. When the largest response current was obtained from WE 1, the sensor head was rotated by 30 degrees to the left. When WE 2 was the most responding sensor, the head was rotated by 15 degrees to the left. Similarly, when WE 3 or WE 4 was the most responding sensor, the sensor head was rotated to the right by 15 or 30 degrees, respectively.

As shown in Figure 10, the chemical source was placed at two different locations: $(x, y)=(110 \mathrm{~mm}, 0 \mathrm{~mm})$ and $(x, y)=$ $(80 \mathrm{~mm}, 50 \mathrm{~mm})$. For each of these source locations, 10 localization trials were conducted. When the sensor head had come within $5 \mathrm{~mm}$ to the chemical source, the trial was regarded as a success and was terminated. When none of the sensors showed above-threshold response for more than $60 \mathrm{~s}$ from the start of the trial, the trial was terminated, as failed. The trial was also regarded as failed when all response currents dropped below the threshold during the search for the chemical source and did not recover for $20 \mathrm{~s}$. The procedure of each trial was as follows:

(1) Start water suction by turning on the pump. 


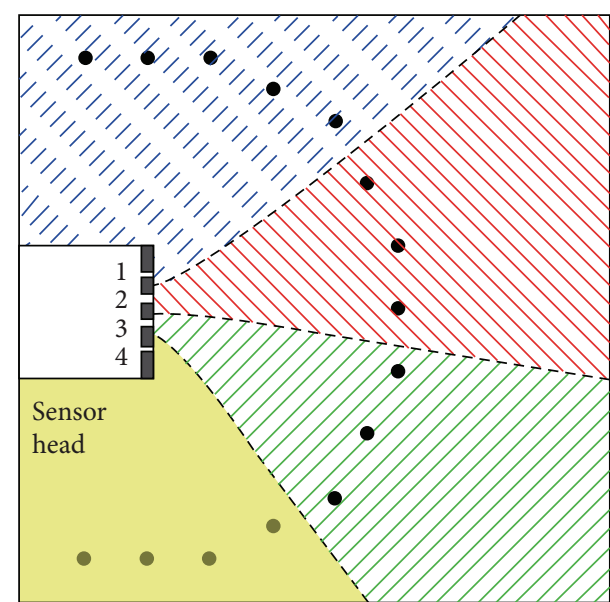

"I", Drawn into slit 1

Drawn into slit 2

Drawn into slit 3

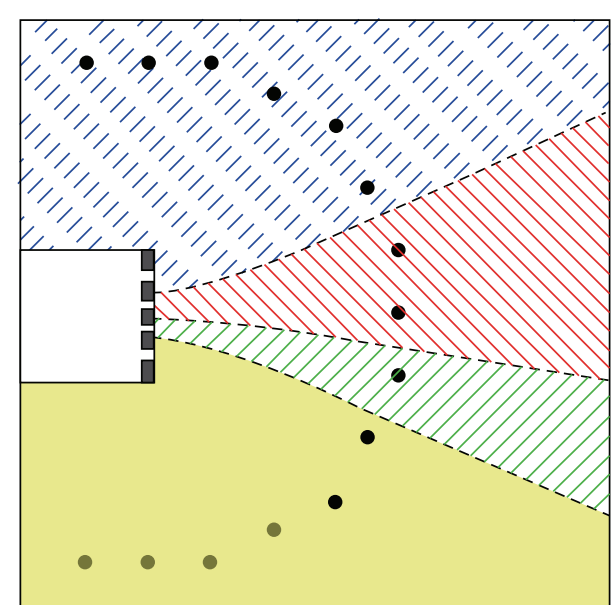

Drawn into slit 4
Drawn into slit 4

E"I, Drawn into slit 1 Drawn into slit 2

Drawn into slit 3

(a)

(b)

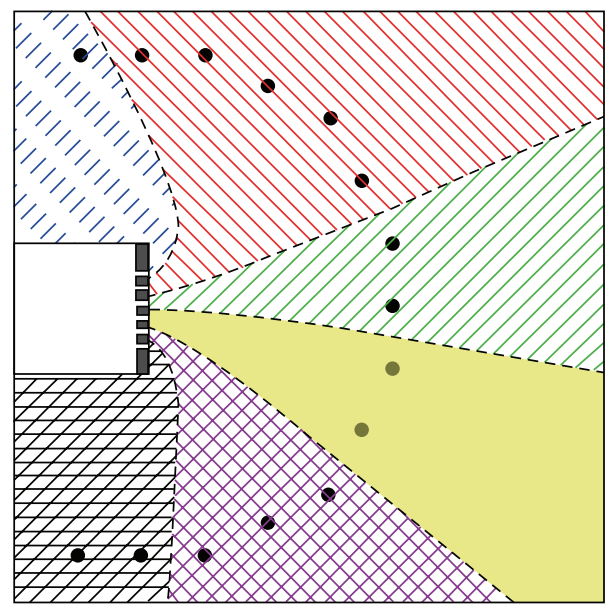

"ㄹㄱ Drawn into slit 1

Drawn into slit 2
Drawn into slit 3

$\square$ Drawn into slit 4

Drawn into slit 5

Drawn into slit 6

(c)

FIGURE 9: Top view of the sensor head and 15 points for chemical release overlaid with maps showing from which area water samples were sucked into each slit. The slits are numbered from the left side of the sensor head. (a)-(c) are the maps for slits (a)-(c), respectively.

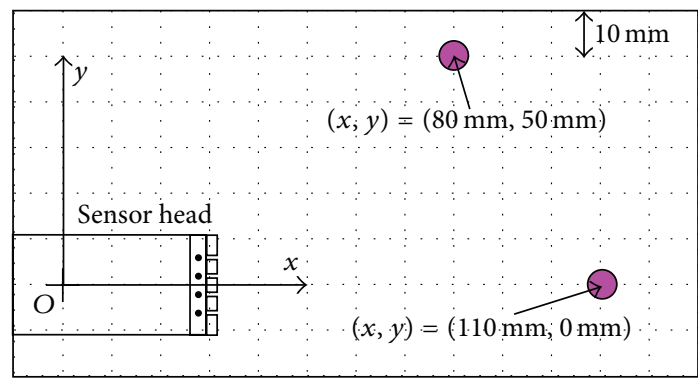

FIGURE 10: Chemical source locations tested in the source localization trials.
(2) Stay until at least one of the sensor response currents exceeds the threshold value.

(3) Rotate the sensor head in the direction of the most responding sensor.

(4) Move the sensor head $5 \mathrm{~mm}$ in that direction.

(5) Repeat steps (2) to (4) until one of the ending conditions described above is met.

A typical result is shown in Figure 11. When the pump was activated, the chemical solution was drawn from the source to the sensor head. When the streak of the chemical 

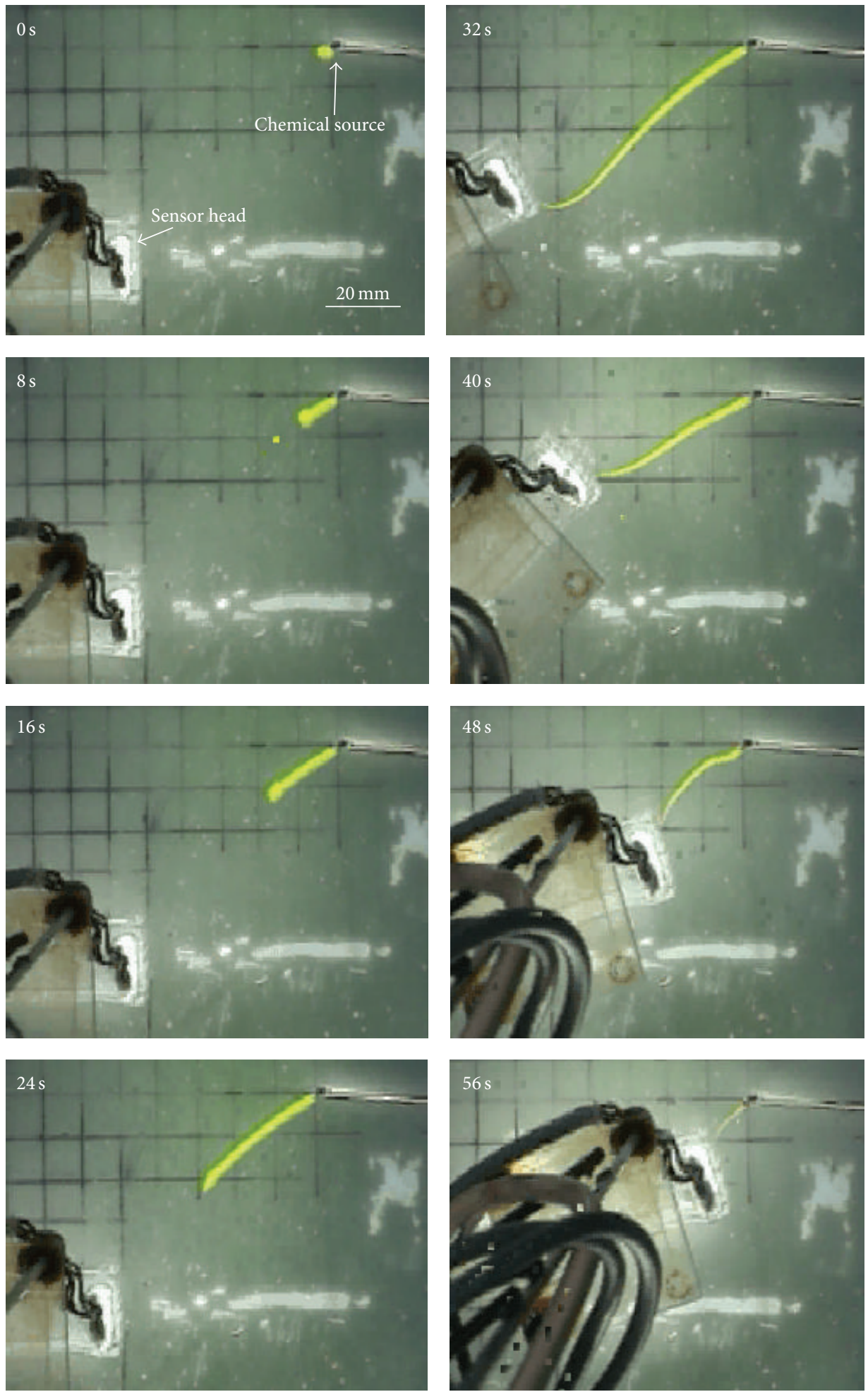

FIGURE 11: Sensor head approaching to the chemical source in a source localization trial.

solution was sucked into one of the slits, the corresponding sensor started to respond. Then, the sensor head started tracking the chemical streak and reached the location of its source. The suction of water samples was relatively slow. It took $30 \mathrm{~s}$ to draw the chemical solution from its source over a distance of $70 \mathrm{~mm}$ to the sensor head. Nevertheless, the active flow generation still had a significant impact on the sensor responses. When the pump was not activated, no response was obtained from the sensors at least for several minutes. In all 20 trials, the chemical solution was 


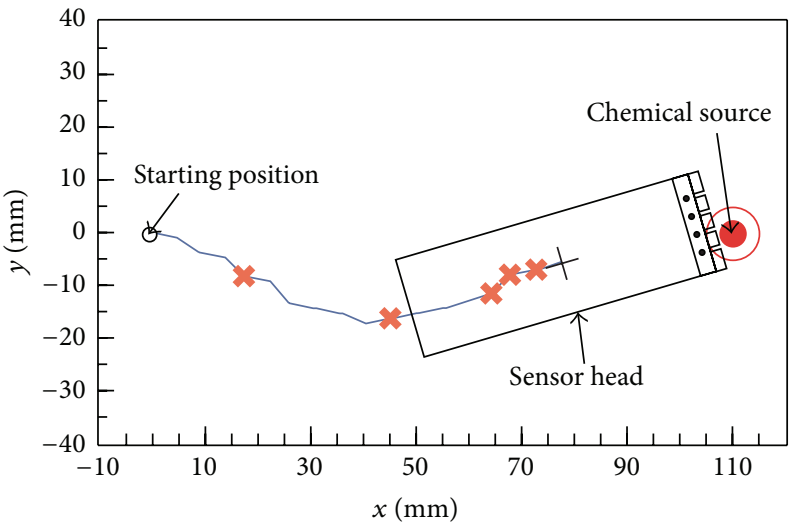

(a)

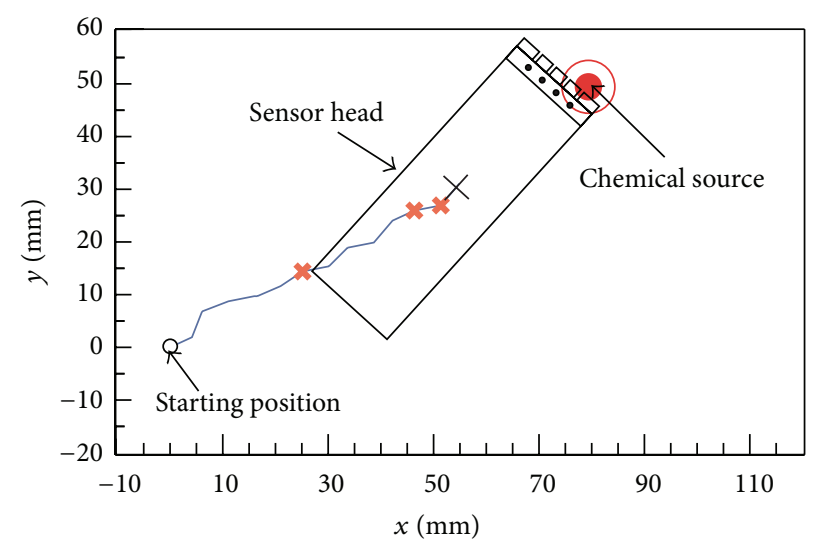

(b)

FIGURE 12: Typical trajectories of the sensor head during the chemical source localization trials. (a) The chemical source was placed at $(x, y)=$ $(110 \mathrm{~mm}, 0 \mathrm{~mm})$. (b) The source was placed at $(x, y)=(80 \mathrm{~mm}, 50 \mathrm{~mm})$. Cross signs show the locations at which the wobbling action was performed.

successfully drawn to the sensors from the two chemical source locations tested. However, the sensor head reached the source location only in $40 \%$ of the trials. In all failed trials, the sensor head started moving toward the source, but it stopped on its way because all sensor response currents dropped below the threshold value and never recovered.

As shown in Figure 11, the chemical solution drawn from the source to the sensor head forms a narrow streak. A smooth laminar flow field was generated by sucking water by the pump. Owing to the flow pattern converging to the slits, the streak of the chemical solution became even narrower as it was drawn to a slit (see, e.g., the picture at $32 \mathrm{~s}$ in Figure 11). It was found from close observation that when the sensor responses were lost, this filamentous streak of the chemical solution was passing between the carbon working electrode and the walls of the slit without touching the electrode surface. As shown in Figure 8, the slits are effective in enhancing the chemical reception at the sensors, but still the reception rate was not $100 \%$. Once the sensors lost contact with the chemical streak, the sensor head was stopped. As long as the sensor head stayed in the same position, the chemical streak was continuously sucked into the same position. Therefore, it was rare to reobtain large sensor response currents before the $20 \mathrm{~s}$ time-out limit.

\section{Chemical Detection Enhancement by Wobbling Action}

The cause of the imperfect chemical reception at the sensors described in the previous section is the extremely narrow chemical streak generated in the smooth laminar flow field. In our previous work [4], almost continuous chemical detection at the sensors was attained by using fanning arms rather than a pump to generate water currents. The fanning motion of the arm introduces fluctuations in the flow field, which causes the chemical streak drawn from the source to meander and enhances the chemical reception at the sensors. Instead, here we propose to make the sensor head intentionally wobble to enhance the chemical reception.

Chemical source localization experiments were performed to test this idea. The experimental setup and conditions were the same as described in the previous section except for the wobbling action of the sensor head. When all sensor responses fell below the threshold, the sensor head was rotated from side to side by \pm 3.5 degrees at $60 \mathrm{deg} / \mathrm{s}$. This wobbling angle was determined to move the sensor electrodes laterally at least by the length of the sensor spacing. Thus, it was ensured that the chemical streak drawn through the small gap between the sensor electrode and the slit wall was made to touch the electrode. The rotational velocity was set to the maximum speed of the stepper motor. The wobbling was continued until at least one of the sensor response currents exceeded the threshold value. When the chemical solution was detected, the sensor head was first returned to the original orientation at the start of the wobbling motion. Then, according to the sensor responses obtained in this returning process, if at least one of the responses was still above the threshold, the sensor head was rotated to the direction of the most responding sensor and was moved in that direction.

Again, 10 chemical source localization trials were conducted for each of the two chemical source locations shown in Figure 10. Pumping was started $5 \mathrm{~s}$ after the start of the chemical release. The chemical source localization was successful in all 20 trials. Figure 12 shows typical trajectories of the sensor head approaching the chemical source. The cross signs in the figure show the positions where the wobbling action was performed. Even when the sensor responses fell below the threshold, the chemical solution was detected again immediately after shaking the sensor head once or twice. When the chemical source was placed at $(x, y)=(110 \mathrm{~mm}$, $0 \mathrm{~mm}$ ), the wobbling action was taken 4.2 times on the way to the chemical source on average in the 10 trials. When the source was placed at $(x, y)=(80 \mathrm{~mm}, 50 \mathrm{~mm})$, the wobbling action was taken 3.0 times on average in the 10 trials. 


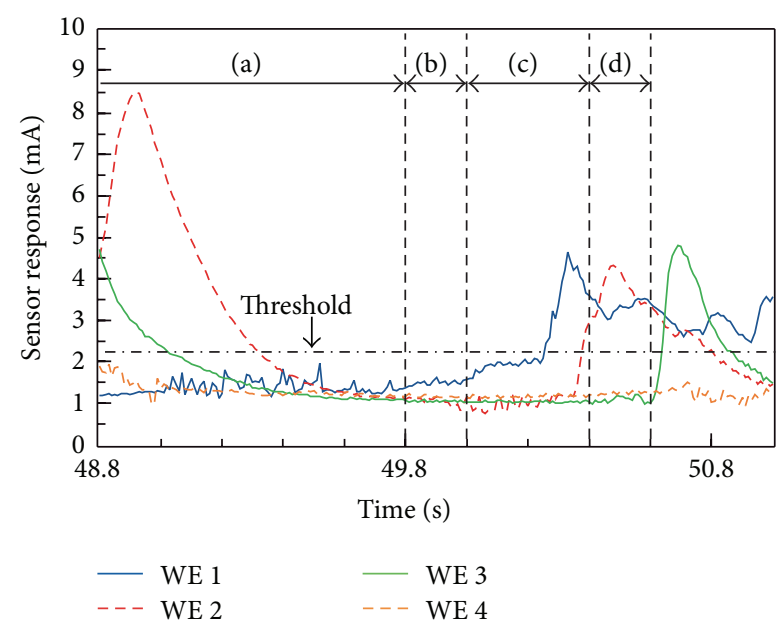

FIGURE 13: Sensor response curves observed in a chemical source localization trial. The contact with the chemical streak was lost at the end of time period (a) as a result of a movement of the sensor head during that period. Since all sensor responses dropped below the threshold value in time period (b), the sensor head was wobbled in period (c). As a result, the above-threshold sensor responses were obtained at the end of (c). In (d), the sensor head was returned to its initial orientation at the start of the wobbling action, and the search for the chemical source was resumed.

Figure 13 shows typical sensor response curves observed during the wobbling motion of the sensor head. In time period (a), the sensor head was moved according to the previously obtained sensor response. Although the system tried to determine the next direction to move in (b), no sensor response exceeded the threshold. Therefore, the translation of the sensor head was stopped and the wobbling motion was started at the end of time period (b). Since no chemical was detected for the first $0.2 \mathrm{~s}$, the wobbling motion was continued for the next $0.2 \mathrm{~s}$ in (c). Since the response current of WE 1 exceeded the threshold from $50.2 \mathrm{~s}$ to $50.4 \mathrm{~s}$, the wobbling motion was stopped in (d) and the sensor head was returned to its original orientation at the start of the wobbling motion. The direction of the chemical source was determined according to the sensor responses obtained in (d), and the translation of the sensor head was resumed. The wobbling motion was also performed at the start of each chemical source localization trial because no sensor response was obtained until the chemical solution was drawn from the chemical source. After the sensor head had left the starting point, the head was shaken 72 times in total in the 20 trials conducted for the two source locations. When the sensor head was shaken, the above-threshold sensor response currents were always obtained. In $90 \%$ of the 72 times, the chemical solution was immediately detected within $1.2 \mathrm{~s}$. The mode value of the duration of the wobbling action was $0.4 \mathrm{~s}$.

In the chemical source localization experiments described here, the response current from each sensor electrode was averaged for every $0.2 \mathrm{~s}$ period, and the direction to move the sensor head was determined by comparing the averaged current values. In contrast, there is experimental evidence that at least some animals estimate the direction of an odor source based on the difference in odor arrival time to the nostrils [18]. Therefore, we looked into the original instantaneous response current values recorded at the $100 \mathrm{~Hz}$ sampling rate. When the pump was activated at the start of each experiment, the chemical solution was sucked into the sensor head, and the sensors started to respond. The difference in the arrival time of the chemical solution to each sensor electrode was in the order of several hundred milliseconds, because of the slow suction velocity as shown in Figure 11. Therefore, when one of the sensors started to respond to an incoming patch of chemical solution, the highest average response current for that $0.2 \mathrm{~s}$ time period was most likely to be obtained from that sensor. In the experiments described in Section 3.1, sensor response curves such as the ones shown in Figure 8 were recorded for all 15 chemical source locations in Figure 5. In all of the 15 measurement runs, the sensor that was the first in responding to the sucked chemical solution showed the highest average response current value for that $0.2 \mathrm{~s}$ time period. In this sense, not only the difference in the intensity of the chemical signal but also the difference in the signal arrival time is reflected to the direction determination in our system. Future work will include trying more sophisticated directional determination algorithms and testing the system under various flow conditions.

\section{Conclusions}

In the work presented in this paper, we investigated the enhancement of chemical reception on underwater chemical sensors by active water sample collection. In our experimental setup, four amperometric electrochemical sensors were aligned in front of an opening of a suction pipe. When a pump connected to the pipe was activated, a smooth laminar flow converging into the pipe opening was generated, and water samples were drawn from the surroundings to the sensors. Our experimental results showed that too smooth suction flow might not be always beneficial for chemical sensing. The streak of the chemical solution drawn to the suction pipe was shaped into a thin filamentous structure and was observed being sucked into the pipe without touching the sensor electrodes. Therefore, slits were placed in front of the sensor electrodes so that a streak of chemical solution drawn from a specific direction was surely guided to the surface of a corresponding sensor electrode. It was shown that the angular detection range of each sensor could be adjusted by changing the width of the corresponding slit. The chemical reception at the sensors was further improved by making the sensing system wobble to force the incoming chemical streak to touch the sensing electrodes. Consequently, a high success rate was achieved in search of a chemical source in a stagnant water aquarium. Although the distance over which water samples can be collected is limited to a few centimeters, active suction has a significant impact on chemical sensing. Future work will include comparing the flow field and the chemical sample collection efficiency of the suction system and those of jet entrainment systems, for example, the crayfish robot [4] and real crayfish. 


\section{Disclosure}

The present address of Hiromi Sakata is Toshiba Corporation.

\section{Conflict of Interests}

The authors declare that there is no conflict of interests regarding the publication of this paper.

\section{References}

[1] F. W. Grasso, T. R. Consi, D. C. Mountain, and J. Atema, "Biomimetic robot lobster performs chemo-orientation in turbulence using a pair of spatially separated sensors: progress and challenges," Robotics and Autonomous Systems, vol. 30, no. 1-2, pp. 115-131, 2000.

[2] F. W. Grasso and J. Atema, "Integration of flow and chemical sensing for guidance of autonomous marine robots in turbulent flows," Environmental Fluid Mechanics, vol. 2, no. 1-2, pp. 95-114, 2002.

[3] W. Li, J. A. Farrell, S. Pang, and R. M. Arrieta, "Moth-inspired chemical plume tracing on an autonomous underwater vehicle," IEEE Transactions on Robotics, vol. 22, no. 2, pp. 292-307, 2006.

[4] M. Ohashi, Y. Kagawa, T. Nakatsuka, and H. Ishida, "Crayfish robot that generates flow field to enhance chemical reception," Journal of Sensor Technology, vol. 2, no. 4, pp. 185-195, 2012.

[5] D. B. Dusenbery, Sensory Ecology: How Organisms Acquire and Respond to Information, W. H. Freeman, New York, NY, USA, 1992.

[6] J. Atema, "Eddy chemotaxis and odor landscapes: exploration of nature with animal sensors," Biological Bulletin, vol. 191, no. 1, pp. 129-138, 1996.

[7] M. J. Weissburg and R. K. Zimmer-Faust, "Odor plumes and how blue crabs use them in finding prey," Journal of Experimental Biology, vol. 197, no. 1, pp. 349-375, 1994.

[8] F. W. Grasso and J. A. Basil, "How lobsters, crayfishes, and crabs locate sources of odor: current perspectives and future directions," Current Opinion in Neurobiology, vol. 12, no. 6, pp. 721-727, 2002.

[9] T. Breithaupt, "Fan organs of crayfish enhance chemical information flow," Biological Bulletin, vol. 200, no. 2, pp. 150-154, 2001.

[10] P. Denissenko, S. Lukaschuk, and T. Breithaupt, "The flow generated by an active olfactory system of the red swamp crayfish," Journal of Experimental Biology, vol. 210, no. 23, pp. 4083-4091, 2007.

[11] J. Yen and J. R. Strickler, "Advertisement and concealment in the plankton: what makes a copepod hydrodynamically conspicuous?" Invertebrate Biology, vol. 115, no. 3, pp. 191-205, 1996.

[12] K. S. Mead, "Inspiration from nature: insights from crustacean chemical sensors can lead to successful design of artificial chemical sensors," in Advances in Chemical Sensors, W. Wang, Ed., pp. 257-276, InTech, Rijeka, Croatia, 2012.

[13] J. P. L. Cox, "Hydrodynamic aspects of fish olfaction," Journal of the Royal Society Interface, vol. 5, no. 23, pp. 575-593, 2008.

[14] P. J. Motta, R. E. Hueter, T. C. Tricas et al., "Functional morphology of the feeding apparatus, feeding constraints, and suction performance in the nurse shark Ginglymostoma cirratum," Journal of Morphology, vol. 269, no. 9, pp. 1041-1055, 2008.
[15] J. Ayers and J. Witting, "Biomimetic approaches to the control of underwater walking machines," Philosophical Transactions of the Royal Society of London A, vol. 365, no. 1850, pp. 273-295, 2007.

[16] T. Kikas, H. Ishida, and J. Janata, "Chemical plume tracking. 3. Ascorbic acid: a biologically relevant marker," Analytical Chemistry, vol. 74, no. 15, pp. 3605-3610, 2002.

[17] G. S. Settles, "Sniffers: fluid-dynamic sampling for olfactory trace detection in nature and homeland security-the 2004 freeman scholar lecture," Journal of Fluids Engineering, vol. 127, no. 2, pp. 189-218, 2005.

[18] J. M. Gardiner and J. Atema, "The function of bilateral odor arrival time differences in olfactory orientation of sharks," Current Biology, vol. 20, no. 13, pp. 1187-1191, 2010. 


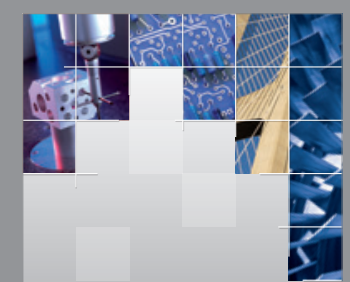

\section{Enfincering}
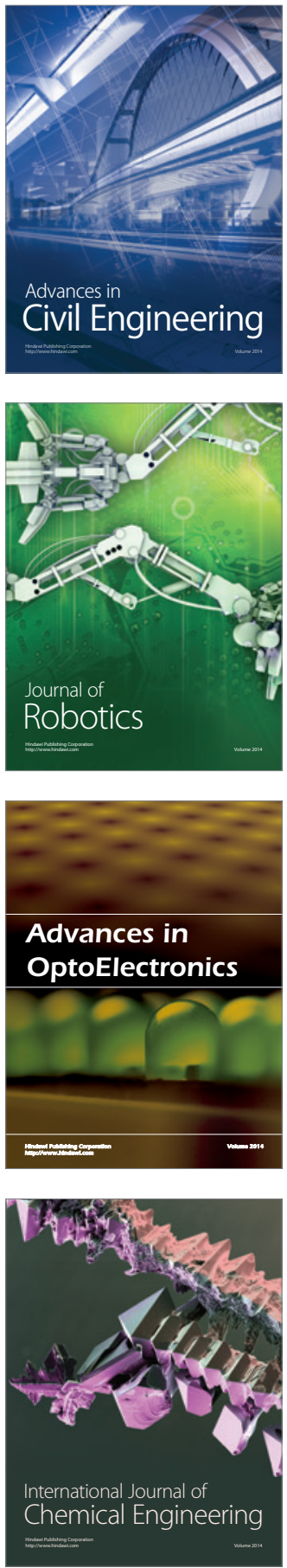

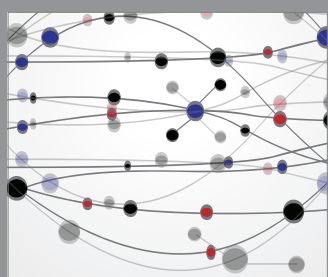

The Scientific World Journal

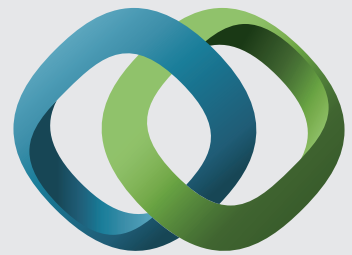

\section{Hindawi}

Submit your manuscripts at

http://www.hindawi.com
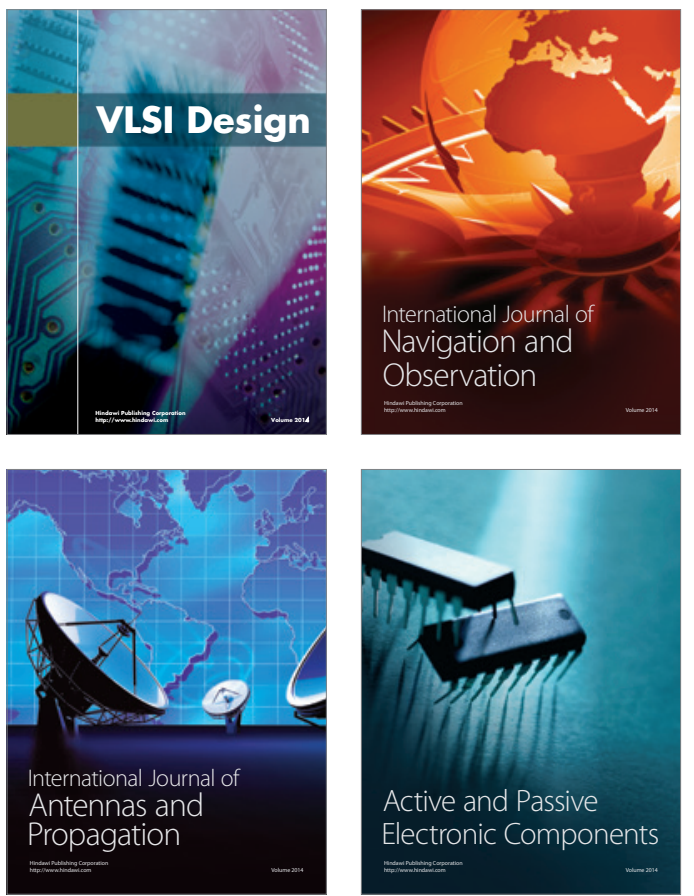
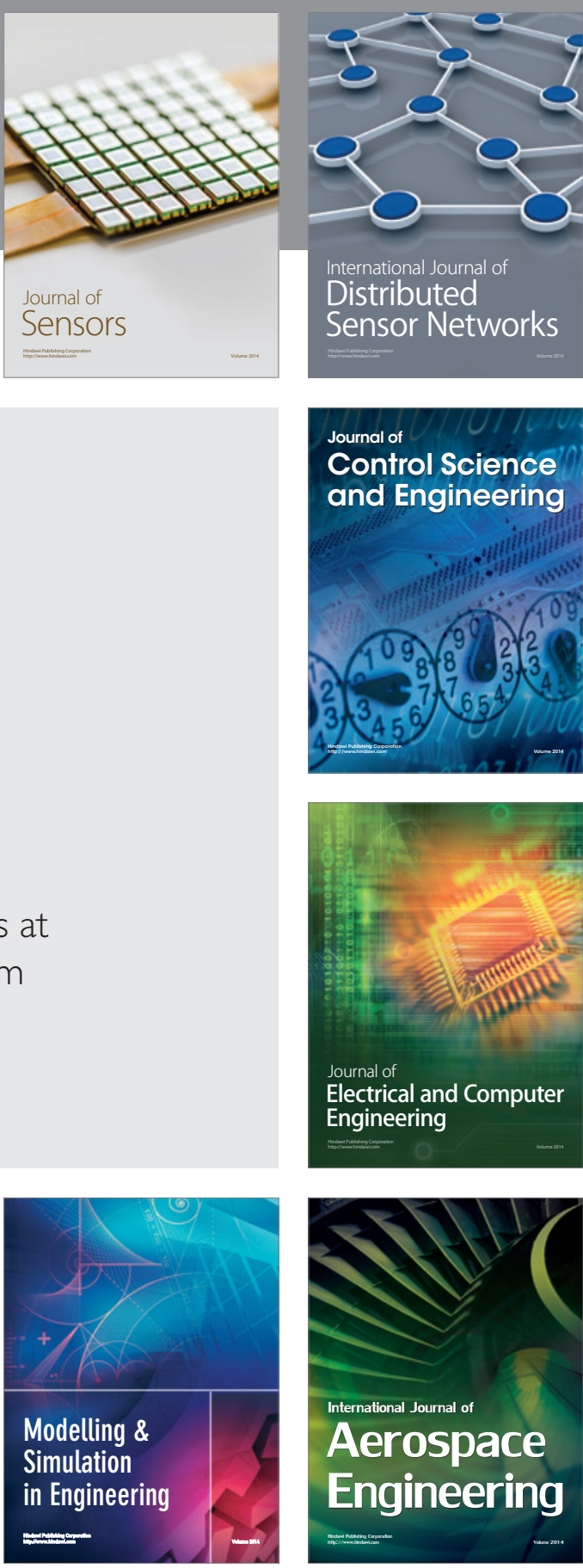

International Journal of

Distributed

Sensor Networks

Journal of

Control Science

and Engineering
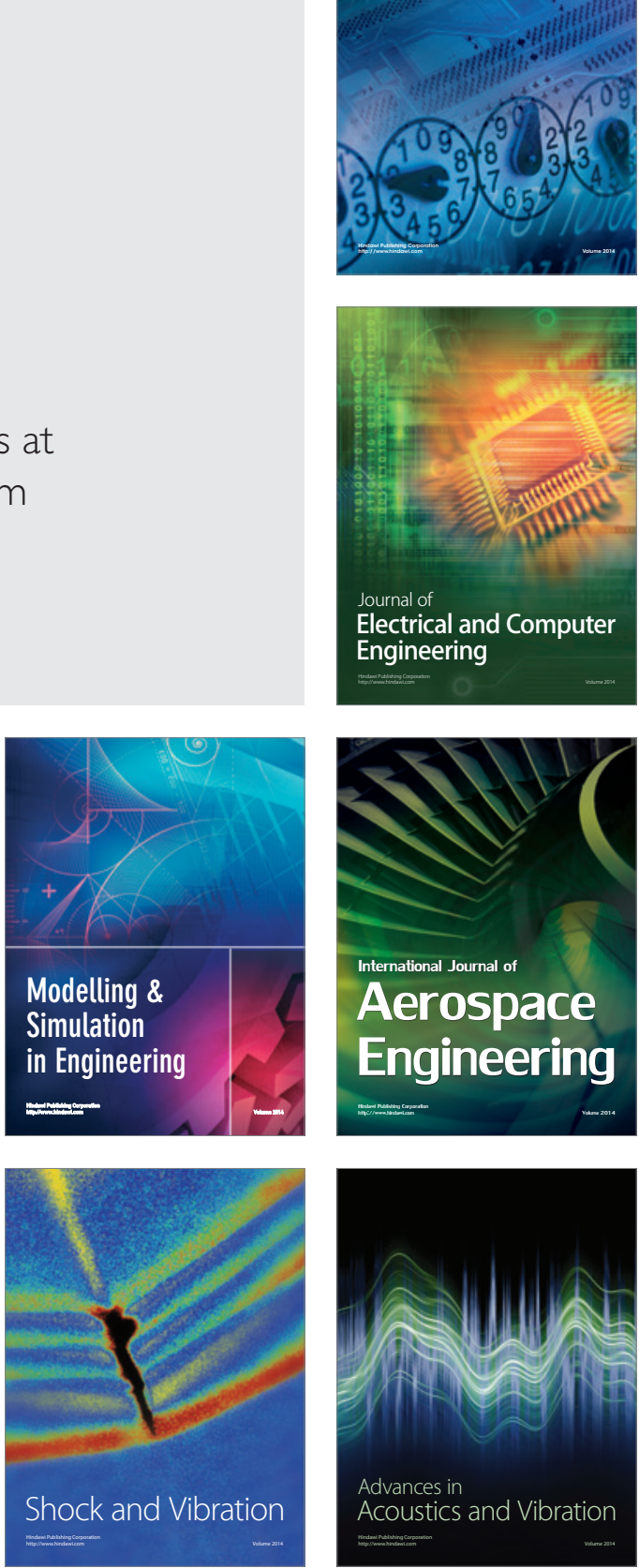